\title{
SPECIAL CNC BASED ON ADVANCED CONTROLLER
}

\author{
Junxi $\mathrm{Bi}^{1,2}$, Tao $\mathrm{Yu}^{1}$, Qiang $\mathrm{Li}^{2}$ \\ ${ }^{\prime}$ CIMS \& Robot Center of Shanghai University, Shanghai University, China; Email: \\ junxibi163@163.com. ${ }^{2}$ College of Mechanical, Inner Mongolia University of Technology, \\ Hohhot, Inner Mongolia, China.
}

\begin{abstract}
This paper introduces a new type of CNC, which is based on Sinumerik 840D and STEP-NC. This is a kernel of $\mathrm{I}^{2} \mathrm{MS}$ (Intelligent integrated Manufacturing System), which includes compute-aided design, computer aided process plan, computer aided manufacturing, and CNC. It provides them a unified platform oriented to $\mathrm{CNC}$ manufacturing, which make their work turned from distributed work to collaborative work. In this paper, we describe and analyze the conceptual framework and key technologies such as $\mathrm{AI}, \mathrm{KM}$, and etc systematically. We also discuss STEP-NC approaches using in CNC.
\end{abstract}

Key words: $\quad$ CNC; STEP-NC; 840D; KM; AI

\section{INTRODUCTION}

Roll grinder is a special machining equipment in the manufacturing industry, it mainly be used to grind some special roll such as CVC roll shape in the steel rolling field. Because of special accuracy requirement of plate shape, thus the grinding accuracy of roll which decided by roll grinder is more and more high. In order to solve this issue, we must adapt higher accuracy $\mathrm{CNC}$ roll grinder. With the development of artificial intelligence, modern CNC machine tools have great changes. But with the continuously increase of the manufacturing system information amount and the lasting improvement of flexibility and automation requirement, the deflection of computer numerical control(CNC) system served in the bottom layer of factory has been shown. It only loyally executed preplanning machining program, the effective apperceive ability and self-control ability are

This project is supported by the Science and Technology Department Foundation of Shanghai Science and Technology Committee under the grant No. 05DZ11C04.

Please use the following format when citing this chapter:

$\mathrm{Bi}$, Junxi, Yu, Tao, Li, Qiang, 2006, in International Federation for Information Processing (IFIP), Volume 207, Knowledge Enterprise: Intelligent Strategies In Product Design, Manufacturing, and Management, eds. K. Wang, Kovacs G., Wozny M., Fang M., (Boston: Springer), pp. 685-690. 
insufficient. There is a trend that advanced manufacturing technologies are to be developed to include numeralization, precision, uttermost, automatization, integration, latticing, intelligentization and green production technologies ${ }^{[1]}$ Manufacturing enterprises are facing serious challenges and pressures from the growing globalization of the economy and the market as well as from the rapid developments of science and technology ${ }^{[2]}$. Small and medium-sized enterprises especially, have to reform their traditional manufacturing methods by using advanced technologies, such as Artificial Intelligence (AI), Knowledge Management (KM), etc, in order to succeed in the increasingly intense competition for markets. Thus, it is of great importance for them to accept the concept of the special computer numerical control based on advanced controller. As the brain for industrial machinery, Computer Numerical Control (CNC) is the core element in modern intelligent integrated manufacturing system $\left(\mathrm{I}^{2} \mathrm{MS}\right)$. The structure of $\mathrm{I}^{2} \mathrm{MS}$ can be shown in figure.1.

In a traditional CNC manufacturing environment, the operation flow usually starts from product design via geometric models created in CAD system, and ends with the generation of machining instructions required to convert raw material into a finished product, based on geometric model. In fact this process includes CAD/CAPP/CAM/CNC/MT. It is an executing mechanism without intelligent and it is a single direction signal workflow in current industrial manufacturing system.

In this paper, a brief conception of special $\mathrm{CNC}$ is presented first, followed by introducing the framework of it based on STEP-NC. Successful implementation in application in roll grinding is given to illustrate how CNC based on $840 \mathrm{D}$ controller works.

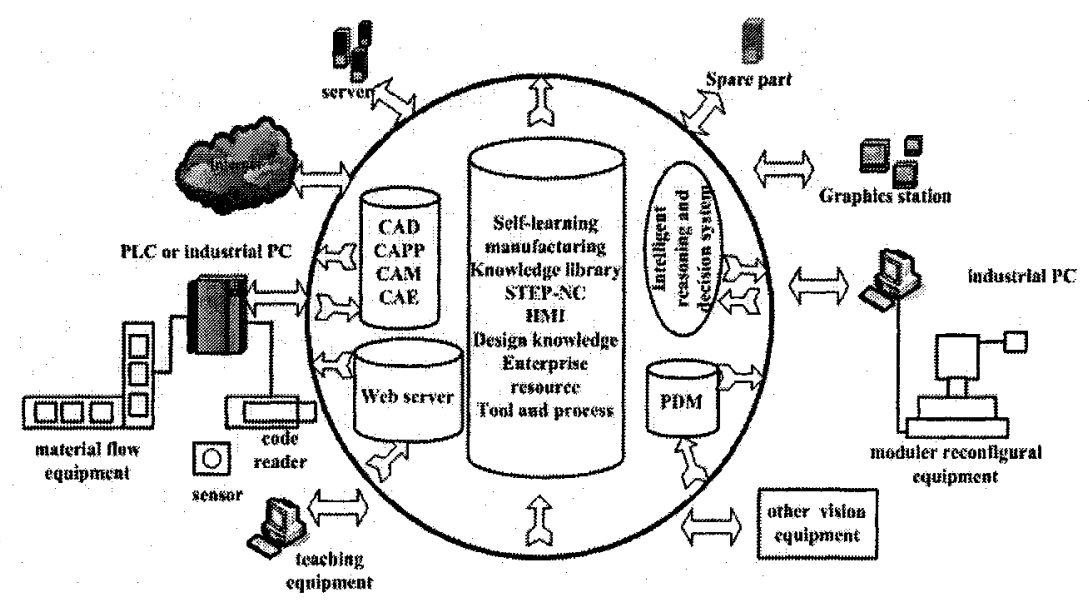

Figure 1. The new model for an $\mathrm{I}^{2} \mathrm{MS}$ 


\section{CONNOTATION OF SPECIAL CNC}

$\mathrm{CNC}$ is the abbreviation of Computer Numerical Control. Here intelligent and integration are two key facts in special CNC system. AI based on the operating principle of the human brain and natural biological evolution, have been received considerable and increasing interest over the past decade and have been applied to CNC machining. Most of the developments are concentrated on an intelligent control algorithm and its application to a traditional CNC machining system, so they have not achieved good results ${ }^{[3}$, ${ }^{4]}$. Various part of system being simply condensed is the idea of intelligent manufacturing model, the system is composed of these condensed intelligent body. Each condensed intelligent body is relatively isolated cell, the system is composed of these cells which can divide and collaborate to work. In order to realize the total intelligence of manufacturing system, it is essential for the intelligence of each cell device. For the intelligence of CNC system, the direction and expression of intelligence is firstly decided, for the integration of CNC system, the method of integration is secondly considered. The intelligence of CNC system is shown as follow ${ }^{[5]}$.

1) The intelligence of job plan

2) The ability of self-learning and improvement

3) The self-adapted man machine interface

4) The intelligent control of manufacturing process

5) The intelligent failure diagnosis

Because the $G$ and $M$ code are employed in the traditional $\mathrm{CNC}$ technology, this has brought some critical problems such as incompatibility between various systems, data sharing and integration, and programming, and so on. Moreover it restricted the open ability and intelligence of CNC system, and led bottle-neck phenomena between the CNC and CAX technology. In order to solve the above-mentioned some reasons, the Europe Union passed a planning of OPTIMAL and provided the concept of STEPNC. STEP-NC (14694) expands STEP into the field of CNC. It redefined a kind of interface between technology planning system of CAM and the control system of NC. This is helpful in decreasing machining time of traditional $\mathrm{CAD} / \mathrm{CAM}$ and in eliminating post processor required in the manufacturing process, and it supports the coming intelligent manufacturing equipments. In fact STEP-NC is a CNC language which has covered the total life cycle of product. That is to say, it is a higher-level programming language for seamless integration of the CAD-CAM-CNC chain. At the present time the research and development of the standard of STEP-NC is still in the laboratory. 840D is an open advanced platform in HMI ,NCK and PLC, thus it is primary task to study and develop open CNC system based on advanced controller and STEP-NC. 
Here CNC means that abstracting some function of traditional CIMS, such as CAD, CAPP, CAM, into a series of modules, which makes the application be divided into a group of tasks frame. The tasks frames could be decomposed into detailed sub-functions. Every function modules is a practical module oriented to manufacturing. The function modules are organized and integrated into the bigger parts modules with the task frame, and these parts modules are integrated into a concrete application CNC system finally with a unified user interface. CNC is also a kind of new philosophy and method in $\mathrm{I}^{2} \mathrm{MS}$.

\section{THE FRAMEWORK OF SPECIAL CNC}

The basic architecture of CNC is composed of:

1) Human-machine interface (HMI)

2) Control function

3) Data processing within $\mathrm{CNC}^{[6]}$

This holds true for $\mathrm{CNC}$ in the architecture presented, the user requirements are implemented in basic architecture in an extended fashion. As shown in figure2, it includes the following main modules:

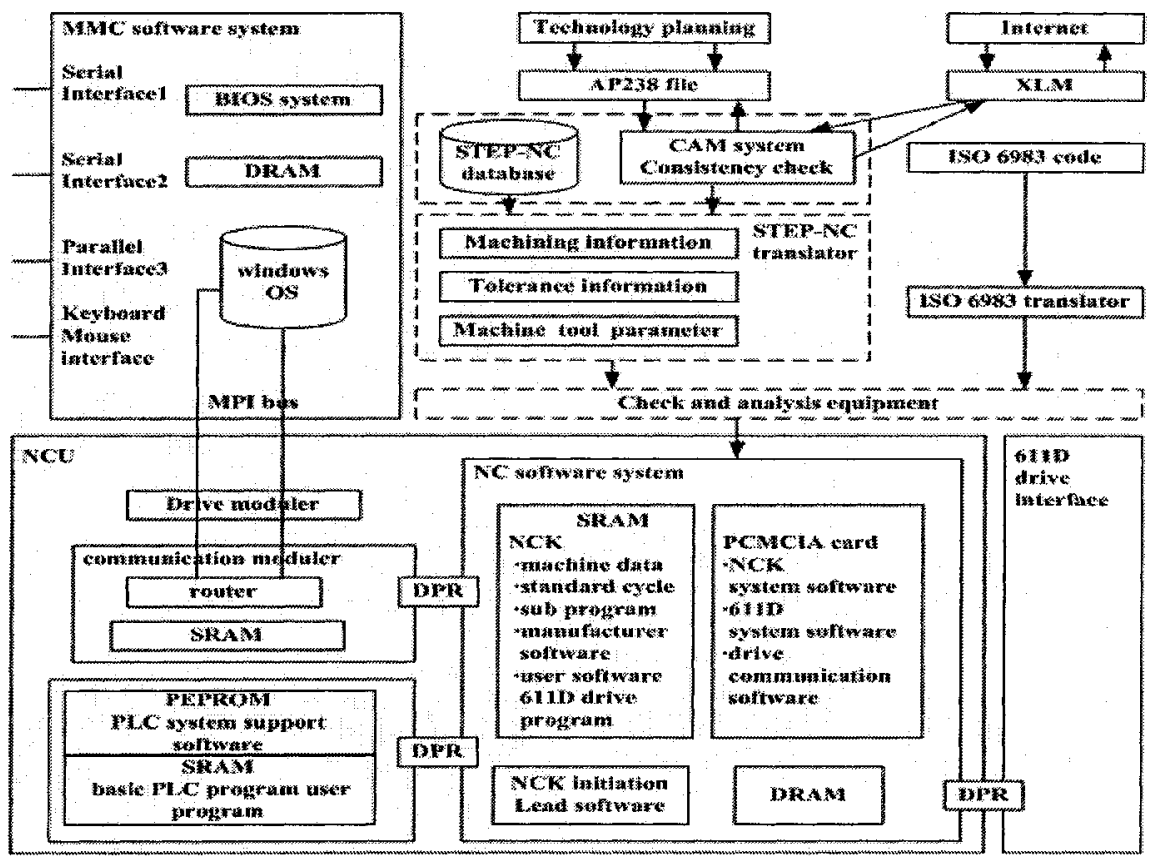

Figure 2. A conceptual architecture of special CNC 
CAD/CAPP/CAM module, which are extended HMI comprehensively covering part programming and tool path generation based on a STEP-NC data model. These modules incorporate the CAD/CAPP/CAM function into the programming system. The standard CAD data (STEP, AP203) can be converted into built-geometric modeling kernel data, by recognizing the machining features and processing these feature information. Output is stored in the machining feature database. CAPP determines the processing sequence and other processing information by using the machining knowledge. Sequentially, CAM extracts NC instruction based on STEP-NC (ISO14649).

NCK/PLC control module which is related to the activities of CNC to carry out successfully the instruction given to it. NCK interprets the tool path commands and executes them by activating the servo mechanism, and PLC executes machinery commands, such as tool change and workpiece loading or unloading. The principal function is to control the machine tool so that the desired shape is produced accurately by the machining operation. Various intelligent control functions are covered here, such as adaptive control, human interaction, quality control, resource management, and selflearning.

Engineering Database based on KM, which provides comprehensive data for the part programming and Tool Path Generation. This database include machining feature database, machining resource database, machining process database, machining knowledge, diagnosis database and so on.

\section{IMPLEMENTATION OF SPECIAL CNC}

We have done researches on special CNC upon Windows NT4.0, Sinumerik $840 \mathrm{D}$ platform. Here we describe working process of special $\mathrm{CNC}$ under the windows-based environment as an example. All the researches and development of Windows NT4.0 based CNC system were based on RG (Roll Grinder).RG is a product of Shanghai Machine Tool Co ltd. RG is used to grind various roll shape in the steel industries. The hardware section of machine is composed of SINUMERIK 840D, industrial PC, MPI (Multiple Point Interface), ProfiBus, and so on.

The software section has an open and modularized structure, which has a standard module interface and each module can be independent with each other and be easily integrated together. One of the important modules is CAD parts module. Self-design CAD of roll shape has been embedded in the software, this module is based on feature, we can perform all the CAD feature function via that interface. Another important module is CAPP intelligent expert process planning system based on knowledge. Here we can 
obtain grinding process parameters easily and we can generate machining instructions based on STEP-NC for the next step via CAM module.

From that illustration above, we can see that special $\mathrm{CNC}$ has integrated some special function of CAD/CAPP/CAM, and all the information are for machining features based on STEP-NC.

\section{CONCLUSION}

This paper discusses a new type of CNC. It provides a unified platform that makes the workflow of project easy to be done. It focuses on some key modules namely CAD/CAPP/CAM/CNC.ISO STEP-NC standard is also introduced and applied in CNC based on sinumerik 840D.

With the development and implementation of STEP-NC and many advanced $\mathrm{AI}, \mathrm{KM}$ etc. technologies in $\mathrm{I}^{2} \mathrm{MS}$, the research and application of $\mathrm{CNC}$ based on advanced controller must be the trend in future $\mathrm{I}^{2} \mathrm{MS}$.

\section{REFERENCES}

1. Shuzi Yang, "Trend in the development of advanced manufacturing technology", Chinese Journal of Mechanical Engineering,Vol 5,No.1,pp.73-77,Dec.2003.

2. Tao Cheng, Jie Zhang, Chunhua Hu, etc. Intelligent Machine Tools in a Distributed Network Manufacturing Mode Environment. International Journal of Advanced Manufacturing Technology,2001,17,pp.221-232.

3. S. Tarng, T. Hwang and Y. S. Wang, "A neural network controller for constant turning force", International Journal of Machine Toolsand Manufacturing, 34(4), pp.453-460, 1994.

4. M. K. Kim, M. W. Cho and K. Kim, "Application of the fuzzy control strategy to adaptive force control of on-minimum phase end milling

5. Shi Xiaojuan, Wang Xiaochun. The modeling Analysis of the Intelligent Numerical Control System, CHINESE JOURNAL OF SCIENTIFIC INSTRUMENT,2003,24(6)

6. SUH S H, CHEON S U.A framework for an intelligent CNC and data model. International Journal of Advanced Manufacturing Technology,2002,19,pp.727-735. 